\title{
Spectropolarimetric study of the classical Cepheid $\eta$ Aql: pulsation and magnetic field
}

\author{
V. Butkovskaya ${ }^{1}$, S. Plachinda ${ }^{1}$, D. Baklanova ${ }^{1}$, and V. Butkovskyi ${ }^{2}$ \\ ${ }^{1}$ Crimean Astrophysical Observatory of Taras Shevchenko National University of Kyiv, \\ 98409, Nauchny, Crimea, Ukraine, \\ email: varya@crao.crimea.ua \\ ${ }^{2}$ Taurida National V. I. Vernadsky University, \\ 95007, Vernadskogo str. 4, Simferopol, Crimea, Ukraine
}

\begin{abstract}
We report the results of spectropolarimetric study of the classical Cepheid $\eta$ Aql. We found that the longitudinal magnetic field of $\eta$ Aql sinusoidally varies with the radial pulsation period, while the amplitude $B$, mean field $B_{0}$, and phases of maximum and minimum field change from year to year. We hypothesize that possible reasons of those variations are stellar axial rotation or dynamo mechanisms.
\end{abstract}

Keywords. stars: magnetic fields, stars: oscillations, stars: individual: $\eta \mathrm{Aql}$

\section{Introduction}

Currently, the question of pulsation modulation of magnetic field in stars, both with convective and radiative envelopes, is still open.

RR Lyr. Babcock (1958) reported a detection of a magnetic field in RR Lyr. The longitudinal component of the field was found to be variable from -1580 to $+540 \mathrm{G}$, but showed no correlation with the pulsation cycle of the star. Romanov et al. $(1987,1994)$ also registered significant magnetic field in RR Lyr, and found the field to be variable with an amplitude of up to $1.5 \mathrm{kG}$ over the pulsation cycle. On the other hand, Preston (1967) and Chadid et al. (2004) detected no convincing evidence of a photospheric magnetic field in the star in the years 1963-1964 and 1999-2002, respectively.

$\eta$ Aql. Photoelectric magnetometer observations of $\eta$ Aql, performed by Borra et al. (1981, 1984), detected no magnetic field in this star. Plachinda (2000) was the first who detected magnetic field on $\eta \mathrm{Aql}$ and reported pulsation modulation of the longitudinal component from -100 to $+50 \mathrm{G}$. Wade et al. (2002) detected no statistically significant longitudinal magnetic field in $\eta$ Aql during 3 nights in 2001 and concluded that $\eta$ Aql is a non-magnetic star, at least at a level of $10 \mathrm{G}$. Grunhut et al. (2010) registered clear Zeeman signatures in Stokes V parameter for $\eta$ Aql and eight other supergiants.

$\gamma$ Peg. Butkovskaya \& Plachinda (2007) reported the modulation of the longitudinal magnetic field in $\beta$ Cephei-type star $\gamma$ Peg (B2 IV) with the amplitude of about 7 G over the 0.15 -day pulsation period of the star.

In order to shed some light on the problem of pulsational modulation of stellar magnetic fields, we continued spectropolarimetric monitoring of $\eta$ Aql during 60 nights between 2002 and 2012 using Coudé spectrograph at the 2.6-m Shajn telescope of the Crimean Astrophysical Observatory (Ukraine). 
Table 1. Parameters of the variability of the longitudinal magnetic field of $\eta$ Aql.

\begin{tabular}{|l|c|c|r|r|r|}
\hline Year & Phase max & Phase min & Amplitude $B, G \mid$ & Mean $B_{0}$, G & F-test \\
\hline 2002 & 0.45 & 0.95 & $12.7 \pm 2.2$ & $5.5 \pm 1.5$ & 0.99 \\
2004 & 0.78 & 0.28 & $13.9 \pm 2.4$ & $4.7 \pm 1.5$ & 0.99 \\
2010 & 0.38 & 0.88 & $4.3 \pm 1.1$ & $-3.1 \pm 1.1$ & 0.98 \\
2012 & 0.60 & 0.10 & $4.2 \pm 2.3$ & $-0.7 \pm 1.5$ & 0.69 \\
\hline
\end{tabular}

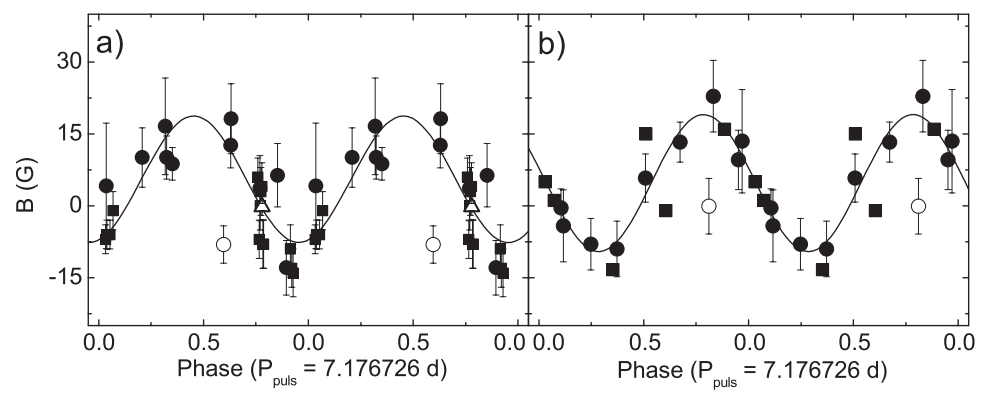

Figure 1. Longitudinal magnetic field of $\eta$ Aql folded with the 7.176726-day pulsation period: a) our 2002 data (closed and open circles), data by Wade et al. (2002) (black squares), and Grunhut et al. (2010) (open triangles); b) our 2004 data (closed and open circles), data by Borra et al. $(1981,1984)$ (black squares). Fitted sinusoids are shown as continuous lines. Open circles represent our data that have not been taken into account in the fits.

\section{Results}

The technique of Zeeman splitting, used for the measurement of the longitudinal magnetic field, is described in detail by Butkovskaya \& Plachinda (2007). We folded all values of the measured longitudinal magnetic field with the pulsational period according to the pulsation ephemeris: JD $=2450100.861+7.176726 E$ (Kiss \& Vinkó 2000), where $E$ is the number of pulsation cycles. As an example, the pulsation modulation of the longitudinal magnetic field of $\eta$ Aql in 2002 and 2004 is illustrated in Fig. 1. We found that the magnetic field sinusoidally varies in phase with the radial pulsation of $\eta$ Aql. This confirms the previous conclusions of Plachinda (2000). However, the amplitude $B$, mean field $B_{0}$, and phases of maximum and minimum field are changing from year to year (see Table 1 where the F-test indicates the statistical reliability of the detected variability for each year). The possible reason for those variations is stellar axial rotation or dynamo mechanisms.

\section{References}

Babcock, H. W. 1958, ApJS, 3, 141

Borra, E. F., Fletcher, J. M., \& Poeckert, R. 1981, ApJ, 247, 569

Borra, E. F., Edwards, G., \& Mayor, M. 1984, ApJ, 284, 211

Butkovskaya, V. \& Plachinda, S. 2007, A\&A, 469, 1069

Chadid, M., Wade, G. A., Shorlin, S. L. S., \& Landstreet, J. D. 2004, A\&A, 413, 1087

Grunhut, J. H., Wade, G. A., Hanes, D. A., \& Alecian, E. 2010, MNRAS, 408, 2290

Kiss, L. L. \& Vinkó, J. 2000, MNRAS, 314, 420

Plachinda, S. I. 2000, A\& A, 360, 642

Preston, G. W. 1967, R. C. Cameron (ed.), The Magnetic and Related Stars (Baltimore: Mono Book Corporation), p. 26

Romanov, Yu. S., Udovichenko, S. N., \& Frolov, M. S. 1987, AZh Lett., 13, 69

Romanov, Yu. S., Udovichenko, S. N., \& Frolov, M. S. 1994, Bull. Spec. Astrophys. Obs., 38, 169

Wade, G. A., Chadid, M., Shorlin, S. L. S., Bagnulo, S., \& Weiss, W. W. 2002, A 6 A, 392, L17 